\title{
Gravitational wave extraction based on Cauchy- characteristic extraction and characteristic evolution
}

\author{
Maria Babiuc $^{1}$, Béla Szilágyi ${ }^{1,2}$, Ian Hawke ${ }^{2,3}$ and Yosef Zlochower ${ }^{4}$ \\ ${ }^{1}$ Department of Physics and Astronomy, University of Pittsburgh, Pittsburgh, PA 15260, USA \\ ${ }^{2}$ Max-Planck-Institut für Gravitationsphysik, Albert-Einstein-Institut, Am Mühlenberg 1, \\ D-14476 Golm, Germany \\ ${ }^{3}$ School of Mathematics, University of Southampton, Southampton SO17 1BJ, UK \\ ${ }^{4}$ Department of Physics and Astronomy, and Center for Gravitational Wave Astronomy, \\ The University of Texas at Brownsville, Brownsville, TX 78520, USA
}

Received 20 September 2005, in final form 17 October 2005

Published 18 November 2005

Online at stacks.iop.org/CQG/22/5089

\begin{abstract}
We implement a code to find the gravitational news at future null infinity by using data from a Cauchy code as boundary data for a characteristic code. This technique of Cauchy-characteristic extraction (CCE) allows for the unambiguous extraction of gravitational waves from numerical simulations. We first test the technique on non-radiative spacetimes: Minkowski spacetime, perturbations of Minkowski spacetime and static black hole spacetimes in various gauges. We show the convergence and limitations of the algorithm and illustrate its success in cases where other wave extraction methods fail. We further apply our techniques to a standard radiative test case for wave extraction, a linearized Teukolsky wave, presenting our results in comparison to the Zerilli technique, and we argue for the advantages of our method of extraction.
\end{abstract}

PACS numbers: $04.25 . \mathrm{Dm}$, 95.30.Sf, 97.60.Lf

\section{Introduction}

The importance of gravitational waveform templates for gravitational wave detectors implies a need for accurate 3D numerical simulations of isolated sources such as binary black hole mergers. These simulations are often done with Cauchy codes based on a ' $3+1$ ' slicing of spacetime. With the slicing conditions most commonly used in numerical simulations, two problems arise: artificial boundary conditions must be placed on the computational domain, and information such as the gravitational news cannot be extracted at future null infinity $\mathcal{I}^{+}$.

To avoid these problems, two possible approaches have been suggested. One way is to evolve the entire spacetime. For example, a hyperboloidal slicing of the spacetime [1-3] allows information to propagate to $\mathcal{I}^{+}$in finite time. Conformal compactifications, such as those suggested by the conformal field equations [4, 5] or employed in [6], allow $\mathcal{I}^{+}$to be 
located at a finite computational coordinate in a regular way. Another example is characteristic evolution based on a Bondi-Sachs line element, which gives a natural description of the radiation region of spacetime extending to $\mathcal{I}^{+}$. Characteristic numerical codes have been used to study tail decay [7], critical phenomena [8-12], singularity structure [13-16] and fluid collapse [17-19], to list just a few examples.

Unfortunately, characteristic methods suffer from the appearance of caustics in the inner strong field region. The problem of caustics can be avoided by evolving the strong field region with a standard Cauchy slicing whilst using the characteristic approach for the exterior. This technique of Cauchy-characteristic matching (CCM) has proved successful in numerical evolution of the spherically symmetric Klein-Gordon-Einstein field equations [20], for 3D non-linear wave equations [21] and for the linearized harmonic Einstein system [22].

The second way of avoiding problems with standard Cauchy codes is a perturbative approach. The standard way of extracting gravitational waves from a numerical simulation is based on perturbation theory, using either the quadrupole formula or first-order gauge invariant formalisms based on the work of Zerilli and Moncrief [23, 24]. The vast majority of waveform templates are currently based upon these approaches. Numerical codes able to solve a generalization of the Zerilli equation to a three-dimensional Cartesian coordinate system and to extract the gravitational signal are reported in [25-28]. Extraction of gravitational waves based on the Zerilli-Moncrief formalism in fully three-dimensional simulations are also presented in [29, 30].

Perturbative methods have also been used to provide boundary conditions at the outer boundary of the Cauchy grid. This approach of Cauchy-perturbative matching has been implemented numerically in [31-33]. All those works are impressive, but discrepancies between the results of a perturbative approach and the full non-linear theory cannot be determined without solving the fully non-linear problem, although error estimates have been made [34].

Of these approaches, CCM has many appealing properties. The characteristic description of the exterior, particularly at $\mathcal{I}^{+}$, allows for a natural extraction of gravitational information such as the news. Matching to a standard Cauchy code in the interior implies that the methods employed in current 3D numerical simulations may immediately be used. As both Cauchy and characteristic approaches have been well tested and commonly used, CCM is a natural way of avoiding the potential problems of caustics in the characteristic region and artificial boundaries in the Cauchy region.

In this work, we take a step towards the full CCM method by using a Cauchy code to provide boundary data for a characteristic code which propagates the solution to $\mathcal{I}^{+}$to extract the waveform. This procedure of Cauchy-characteristic extraction (CCE) allows the computation of gravitational waves in an unambiguous fashion. CCE was successfully implemented in the quasispherical approximation in [35]; here we demonstrate it in the fully non-linear case.

In this work, the outer region extending to $\mathcal{I}^{+}$is numerically evolved by the Pitt null code [20, 35-42]. The link between the Cauchy and the characteristic modules is done by a non-linear 3D CCE algorithm [35, 41-44]. At the outer edge of the characteristic grid, the Bondi news is computed [37, 39, 45, 46]. We have imported the CCE code into the Cactus computational infrastructure [47-50]. Within this infrastructure, we have been able to use two separate Cauchy codes implementing the BSSN and harmonic $3+1$ formulations of the Einstein equations. This allows us to test the robustness of the CCE approach.

We compare CCE with the Zerilli extraction technique [23, 24, 51] for both non-radiative and radiative spacetimes. The Zerilli formalism is based on the paper of Nagar and Rezzolla [52] which reviews and collects the relevant expressions related to the Regge-Wheeler and 
Zerilli equations for the odd- and even-parity perturbations of a Schwarzschild spacetime. The conventions presented in their review are implemented in the Wave Extract code, included in the Cactus computational toolkit open source infrastructure [49].

For non-radiative spacetimes, the comparison gives results consistent with [34]: the CCE algorithm is $\mathcal{O}\left(\Delta^{2}\right)$ accurate (where $\Delta$ is the computational grid step), while the accuracy of the perturbative (Zerilli) approach is $\mathcal{O}\left(r^{-2}\right)$ where $r$ is the radius of the wave extraction sphere. Since the signal is $\mathcal{O}\left(r^{-1}\right)$, this implies an $\mathcal{O}\left(r^{-1}\right)$ relative error in the Zerilli approach. As a result, it is shown in [34] that CCM is more efficient computationally in the sense that, as the desired error goes to zero, the amount of computation required for CCM becomes negligible compared to a pure Cauchy computation.

We further apply our algorithms to the study of the propagation of a linearized Teukolsky gravitational wave [27, 53-55] and we compare the CCE and Zerilli wave signal. For the case of a large extraction radius, we find that both methods give very good results and we demonstrate convergence to the analytical waveform of the Teukolsky solution for the CCE news. We show that the CCE waveform does not depend upon the extraction radius, which is a major advantage of the CCE method. A small extraction radius has no effect on the CCE waveform but it introduces errors in the Zerilli waveform.

In section 2, we outline some general background and notation. In section 3, we detail the transformation from the Cauchy slice to the characteristic slice. In section 4.1, we give a brief summary of the characteristic evolution code. In section 4.2, we describe how the news is computed at $\mathcal{I}^{+}$. Finally, in section 5, we give two sets of tests in full 3D numerical relativity to validate the accuracy, convergence and robustness of the CCE algorithm. The first set contains four non-radiative tests, with no gravitational wave content. The second one is a radiative three-dimensional test. The results show that CCE is valid and accurate in both non-radiative and truly radiative situations.

\section{Notation, geometry and metrics}

The implementation of CCE described here follows previous descriptions of Cauchycharacteristic extraction and matching in the literature. Much of the work has been presented earlier [35, 41-44]. Here we briefly outline the notation, geometry and metrics used.

The geometry is described by two separate foliations, neither of which cover the entire spacetime. The Cauchy foliation is described using a standard $3+1$ ADM-type metric [56],

$$
\mathrm{d} s^{2}=-\left(\alpha^{2}-\beta_{i} \beta^{i}\right) \mathrm{d} t^{2}+2 \beta_{i} \mathrm{~d} t \mathrm{~d} x^{i}+\gamma_{i j} \mathrm{~d} x^{i} \mathrm{~d} x^{j} .
$$

Many different formulations can be used, given initial and boundary data, to evolve the 3-metric $\gamma_{i j}$. In the following, we shall consider either the BSSN formulation $[57,58]$ as implemented in [59] or the generalized harmonic formulation [60] as implemented in the Abigel code [61]. In both cases, all interaction between the Cauchy and characteristic foliations will be performed in terms of the ADM metric, equation (2.1).

The Cauchy slice does not extend to asymptotic infinity. Instead, an artificial boundary is placed at $\left|x^{i}\right|=L^{i}$. Within this artificial boundary, a world-tube $\Gamma$ is constructed such that its intersection with any $t=$ const Cauchy slice is defined as a Cartesian sphere $x^{2}+y^{2}+z^{2}=R^{2}$, with angular coordinates labelled by $\tilde{y}^{\tilde{A}}, \tilde{A}=(2,3)$. The world-tube $\Gamma$ is then used as the inner boundary of a characteristic foliation which uses the standard Bondi-Sachs metric $[62,63]$

$\mathrm{d} s^{2}=-\left(\mathrm{e}^{2 \beta} \frac{V}{r}-r^{2} h_{A B} U^{A} U^{B}\right) \mathrm{d} u^{2}-2 \mathrm{e}^{2 \beta} \mathrm{d} u \mathrm{~d} r-2 r^{2} h_{A B} U^{B} \mathrm{~d} u \mathrm{~d} y^{A}+r^{2} h_{A B} \mathrm{~d} y^{A} \mathrm{~d} y^{B}$. 
Here $u$ labels the outgoing null hypersurfaces, $y^{A}=\tilde{y}^{\tilde{A}}$ the angular coordinates (the null rays emanating from the world-tube) and $r$ is a radial surface area distance. The angular metric $h_{A B}$ obeys the condition

$$
\operatorname{det}\left(h_{A B}\right)=\operatorname{det}\left(q_{A B}\right),
$$

where $q_{A B}$ is the unit-sphere metric. This coordinate system consistently covers the world-tube $\Gamma$ and the exterior spacetime as long as it is free of caustics.

The free variables in the Bondi-Sachs metric are then $V, \beta, U^{A}$ and $h_{A B}$. The physical interpretation of these variables is that $h_{A B}$ contains the two radiative degrees of freedom, $\mathrm{e}^{2 \beta}$ measures the expansion of the null cone and $V / r$ is the counterpart of the Newtonian gravitational potential. The $2+1$ decomposition of the intrinsic metric on the $r=$ const world-tube

$$
\gamma_{i j} \mathrm{~d} y^{i} \mathrm{~d} y^{j}=-\mathrm{e}^{2 \beta} \frac{V}{r} \mathrm{~d} u^{2}+r^{2} h_{A B}\left(\mathrm{~d} y^{A}-U^{A} \mathrm{~d} u\right)\left(\mathrm{d} y^{B}-U^{B} \mathrm{~d} u\right)
$$

identifies $r^{2} h_{A B}$ as the intrinsic 2-metric of the $u$ foliation, $-U^{A}$ as the shift vector and $\mathrm{e}^{2 \beta} V / r$ as the square of the lapse.

\section{The CCE algorithm}

The crucial task of the CCE algorithm is to take Cauchy data given in the ADM form (2.1) in a neighbourhood of the world-tube $\Gamma$ and to transform it into boundary data for the BondiSachs metric (2.2). Then, the Bondi code can use the hypersurface equations to evolve the appropriate quantities out to $\mathcal{I}^{+}$so that the gravitational news may be extracted there. Much of the present version of the CCE algorithm has been presented in earlier work [35, 41-44], so in this section we will give a brief description, highlighting a few new features.

In section 2, the world-tube was defined as a Cartesian sphere $x^{2}+y^{2}+z^{2}=R^{2}$, with angular coordinates labelled by $\tilde{y}^{\tilde{A}}, \tilde{A}=(2,3)$. In addition to the angular coordinates, we set $u=t$ on the world-tube and choose the fourth coordinate, $\lambda$, to be the affine parameter along the radial direction, with $\lambda_{\mid \Gamma}=0$. The characteristic cones are constructed such that $\lambda$ is the future oriented, outgoing null direction normal to the foliation of $\Gamma$. (In order to avoid a singular Jacobian for the Cauchy-characteristic coordinate transformation, we require that the world-tube $\Gamma$ be timelike.) The affine parameter $\lambda$ is used because the world-tube is not constructed to be a surface of constant Bondi $r$.

The choice of the angular coordinates is determined following [64] by the use of two stereographic patches. The use of two patches avoids numerical difficulties in taking derivatives near the poles when standard spherical coordinates are used. The use of only two patches may not give the most accurate numerical results; various ways of discretizing the 2-sphere on multiple patches are discussed in [65]. The two patches are centred around the North and South poles with the stereographic coordinates related to the usual spherical coordinates $(\theta, \phi)$ by

$$
\xi_{\text {North }}=\sqrt{\frac{1-\cos \theta}{1+\cos \theta}} \mathrm{e}^{\mathrm{i} \phi}, \quad \xi_{\text {South }}=\sqrt{\frac{1+\cos \theta}{1-\cos \theta}} \mathrm{e}^{-\mathrm{i} \phi}
$$

and $\xi=\tilde{y}^{2}+\mathrm{i} \tilde{y}^{3}=q+\mathrm{i} p$, where $\mathrm{i}=\sqrt{-1}$. We also introduce the complex vector on the sphere $q^{A}=(P / 2)\left(\delta_{2}^{A}+\mathrm{i} \delta_{3}^{A}\right)$ and its co-vector $q_{A}=(2 / P)\left(\delta_{A}^{2}+\mathrm{i} \delta_{A}^{3}\right)$, with $P=1+\xi \bar{\xi}=1+q^{2}+p^{2}$. The orthogonality condition $\bar{q}_{A} q^{A}=2$ is satisfied by construction. The unit-sphere metric corresponding to these coordinates is

$$
q_{A B}=\frac{1}{2}\left(q_{A} \bar{q}_{B}+\bar{q}_{A} q_{B}\right)=\frac{4}{P^{2}}\left[\begin{array}{ll}
1 & 0 \\
0 & 1
\end{array}\right] .
$$


The angular subspace in the Bondi code is treated by use of the eth formalism and spin-weighted quantities. (See [39, 64] for details.)

The CCE algorithm starts by interpolating the Cauchy metric $\gamma_{i j}$, lapse $\alpha$ and shift $\beta^{i}$ and their spatial derivatives onto the world-tube. Time derivatives are computed via backwards finite differencing done along $\Gamma$, e.g., at $t=t_{N}$ we write

$$
\left(\partial_{t} F\right)_{[N]}=\frac{1}{2 \Delta t}\left(3 F_{[N]}-4 F_{[N-1]}+F_{[N-2]}\right)+\mathcal{O}\left((\Delta t)^{2}\right) .
$$

Knowledge of the Cauchy metric and its 4-derivative is enough to compute the affine metric $\tilde{\eta}^{\tilde{\alpha} \tilde{\beta}}$ as a Taylor series expansion around the world-tube

$$
\tilde{\eta}^{\tilde{\alpha} \tilde{\beta}}=\left.\tilde{\eta}^{\tilde{\alpha} \tilde{\beta}}\right|_{\Gamma}+\left.\lambda \tilde{\eta}_{, \lambda}^{\tilde{\alpha} \tilde{\beta}}\right|_{\Gamma}+\mathcal{O}\left(\lambda^{2}\right) \text {. }
$$

Next, a second null coordinate system (the Bondi-Sachs system) is introduced

$$
y^{\alpha}=\left(y^{1}, y^{A}, y^{4}\right), \quad \text { where } \quad y^{A}=\tilde{y}^{\tilde{A}}, \quad y^{1}=\tilde{y}^{1}=u .
$$

The fourth coordinate $y^{4}=r$ is a surface area coordinate, defined by

$$
r=\left(\frac{\operatorname{det}\left(\tilde{\eta}_{\tilde{A} \tilde{B}}\right)}{\operatorname{det}\left(q_{A B}\right)}\right)^{\frac{1}{4}}=\frac{P}{2} \operatorname{det}\left(\tilde{\eta}_{\tilde{A} \tilde{B}}\right)^{\frac{1}{4}} .
$$

The Bondi-Sachs metric on the extraction world-tube can be computed as

$$
\eta_{\mid \Gamma}^{\mu v}=\left(\frac{\partial y^{\mu}}{\partial \tilde{y}^{\tilde{\alpha}}} \frac{\partial y^{v}}{\partial \tilde{y}^{\tilde{\beta}}} \tilde{\eta}^{\tilde{\alpha} \tilde{\beta}}\right)_{\mid \Gamma} .
$$

Note that the metric on the sphere is unchanged by this coordinate transformation, i.e., $\eta^{A B}=\tilde{\eta}^{\tilde{A} \tilde{B}}$. Therefore, one only needs to work with the Jacobian components that correspond to derivatives of $r$.

In terms of $q^{A}, \bar{q}^{A}$, the two-dimensional metric $\eta^{A B}$ can be encoded into the metric functions

$$
J \equiv \frac{1}{2} q^{A} q^{B} h_{A B}, \quad K \equiv \frac{1}{2} q^{A} \bar{q}^{B} h_{A B} .
$$

The determinant condition (2.3) translates into

$$
K^{2}=1+J \bar{J} .
$$

With $h_{A B}$ symmetric and of fixed determinant, there are only two degrees of freedom in the angular metric that are encoded into the complex function $J$.

From equation (2.2), we have

$$
\beta=-\frac{1}{2} \log \left(-\eta^{r r}\right)=\frac{1}{2} \log (r, \lambda) .
$$

This quantity is a measure of the expansion of the light rays as they propagate outwards. The CCE and the Bondi codes have been implemented with the assumption that $r_{, \lambda}>0$ (or that $\beta$ is real).

The radial-angular components $\eta^{r A}$ can be represented by

$$
U \equiv U^{A} q_{A}=\frac{\eta^{r A}}{\eta^{r u}}
$$

while the radial-radial component $\eta^{r r}$ is contained in

$$
W \equiv \frac{V-r}{r^{2}} .
$$

In addition to these quantities, in [40] the auxiliary variables

$$
v \equiv \bar{\varnothing} J=\frac{1}{2} h_{A B, C} q^{A} q^{B} \bar{q}^{C},
$$




$$
\begin{aligned}
& k \equiv \succsim K=\frac{1}{2} h_{A B, C} q^{A} \bar{q}^{B} q^{C}+2 \xi K, \\
& B \equiv \succsim \beta=\beta{ }_{, A} q^{A}
\end{aligned}
$$

have been introduced to eliminate the need to explicitly use second angular derivatives in the Bondi evolution code. The required boundary data are $J, \beta, U, \partial_{r} U, W, v, k$ and $B$. (See section 4.1.) Note that once the Bondi-Sachs metric is known on the world-tube, one can only obtain $J, \beta, U, W$. In order to provide the rest of the necessary boundary data, we need the radial derivative of the Bondi-Sachs metric

$$
\left(\partial_{\lambda} \eta^{\mu \nu}\right)_{\mid \Gamma}=\left(\frac{\partial^{2} y^{\mu}}{\partial \lambda \partial \tilde{y}^{\tilde{\alpha}}} \frac{\partial y^{v}}{\partial \tilde{y}^{\tilde{\beta}}} \tilde{\eta}^{\tilde{\alpha} \tilde{\beta}}+\frac{\partial y^{\mu}}{\partial \tilde{y}^{\tilde{\alpha}}} \frac{\partial^{2} y^{v}}{\partial \lambda \partial \tilde{y}^{\tilde{\beta}}} \tilde{\eta}^{\tilde{\alpha} \tilde{\beta}}+\frac{\partial y^{\mu}}{\partial \tilde{y}^{\tilde{\alpha}}} \frac{\partial y^{v}}{\partial \tilde{y}^{\tilde{\beta}}} \tilde{\eta}_{, \lambda}^{\tilde{\alpha} \tilde{\beta}}\right)_{\mid \Gamma} .
$$

With $\tilde{\eta}_{, \lambda}^{\tilde{\alpha} \tilde{\beta}}$ already known, the only non-trivial parts in equation (3.16) are the Jacobian terms

$$
r_{, \lambda \tilde{\alpha}}=\frac{\partial^{2} y^{1}}{\partial \lambda \partial \tilde{y}^{\tilde{\alpha}}} .
$$

These depend on the second derivatives of the Cauchy metric. In order to avoid possible numerical problems caused by interpolating second derivatives onto the world-tube, we calculate $r_{, \lambda \tilde{A}}$ by taking centred derivatives of $r_{, \lambda}$ on the world-tube and we calculate $r_{, \lambda u}$ by backwards differencing in time along the world-tube. The remaining term $r_{, \lambda \lambda}$ is calculated using the identity

$$
\beta_{, \lambda}=-\frac{r_{, \lambda \lambda}}{2 r_{, \lambda}}
$$

and the characteristic equation

$$
\beta_{, r}=\frac{r}{8}\left(J_{, r} \bar{J}_{, r}-\left(K_{, r}\right)^{2}\right) .
$$

(The right-hand side of equation (3.19) can be computed in terms of $J_{, r}=J_{, \lambda} / r_{, \lambda}$, which, in turn, can be computed in terms of already known quantities.)

Knowledge of the radial derivative of the Bondi metric is important not only for obtaining $\left(\partial_{r} U\right)_{\mid \Gamma}$ but also because the grid structure of the Bondi code is based on the radial coordinate $r$, and the extraction world-tube will not, in general, coincide with any of these radial grid points. We need to use, therefore, Taylor series expansions to fill the Bondi grid points surrounding $\Gamma$ with the necessary boundary data. We write, e.g.,

$$
\beta=\beta_{\mid \Gamma}+\lambda \beta_{, \lambda}+\mathcal{O}\left(\lambda^{2}\right)
$$

Another problem is the need to provide the auxiliary angular variables, $B, k$ and $v$ on the world-tube. These have been defined as the $ð$ derivatives of Bondi fields in the $y^{\alpha}$ frame, while taking angular derivatives on the world-tube amounts to computing $ð$ derivatives in the $\tilde{y}^{\tilde{\alpha}}$ frame. The correction term between the two frames is

$$
ð F=\tilde{\delta} F-\frac{F_{, \lambda}}{r_{, \lambda}} \tilde{\delta} r .
$$

The quantities $J, \tilde{\partial} J$ and $J_{, \lambda}$ are known from the interpolated Cauchy data, while $J_{, \lambda \lambda}$ is not computed in CCE. Thus, one can compute $ð J$ but not $\delta J_{, \lambda}$. As a consequence, we cannot use a simple Taylor series expansion to place $v$ on the Bondi grid to $\mathcal{O}\left(\lambda^{2}\right)$ accuracy. The solution to this problem is to first place $J$ on Bondi grid points surrounding the world-tube, then compute $\Varangle J$ on those points (i.e., compute angular derivatives in the Bondi-Sachs frame), and then calculate $\partial_{\lambda} \partial J$ on the world-tube by use of the neighbouring Bondi grid values of $ð J$. With $v=ð J$ and its radial derivative known on the world-tube, we can then use the standard $\mathcal{O}\left(\lambda^{2}\right)$ expansion to provide $v$ at Bondi grid points surrounding the world-tube. This way we make maximal use of the Cauchy data as interpolated onto the world-tube and minimal use of the finite-difference $ð$ algorithm that has its own discontinuous $\mathcal{O}\left(\Delta^{2}\right)$ error at the edges of the stereographic patches. A similar approach is used for $k=ð K$ and $B=ð \beta$. 


\section{The Bondi code and the news algorithm}

\subsection{The Bondi code}

The inner workings of the Bondi code had been described in detail elsewhere (see [20, 35-42]) so here we give only a brief overview of the algorithm. As already stated in section 3 , the variables of the code are $J, \beta, U, W$ as well as $v, k, B$. Out of these, the equation for $J$ is the only one to contain a time derivative. For this reason, $J$ is updated via an evolution stencil that involves two time levels. The rest of the variables are integrated radially from the world-tube out to $\mathcal{I}^{+}$. The integration constants are set by CCE. All of these radial integration equations are first differential order in $r$ except for the $U$ equation which contains $U_{, r r}$. For this reason, integrating $U$ requires two constants, which explains the need to provide, at the world-tube, $U$ as well as $U_{, r}$.

\subsection{The news algorithm}

The calculation of the Bondi news function on $\mathcal{I}^{+}$is based on the algorithm developed in [39] with the modifications introduced in [45, 46] (an alternative calculation for the news was recently introduced in [66]). Here we present an overview of the algorithm.

The Bondi-Sachs metric (2.2) in a neighbourhood of $\mathcal{I}^{+}$in $\left(u, x^{A}, l=1 / r\right)$ coordinates (after multiplying by a conformal factor $l^{2}$ ) has the form

$$
l^{2} \mathrm{~d} s^{2}=\mathcal{O}\left(l^{2}\right) \mathrm{d} u^{2}+2 \mathrm{e}^{2 \beta} \mathrm{d} u \mathrm{~d} l-2 h_{A B} U^{B} \mathrm{~d} u \mathrm{~d} x^{A}+h_{A B} \mathrm{~d} x^{A} \mathrm{~d} x^{B},
$$

where the metric variables $\beta, U^{A}$ and $h_{A B}$ have the asymptotic expansions $\beta=H+$ $\mathcal{O}\left(l^{2}\right), U^{A}=L^{A}+\mathcal{O}(l)$ and $h_{A B}=H_{A B}+l c_{A B}+\mathcal{O}\left(l^{2}\right)$. We can always find coordinates $\left(u_{B}, q_{B}, p_{B}, l_{B}\right)$ (hereafter referred to as 'inertial' coordinates), and an associated conformal metric $\mathrm{d} s_{B}{ }^{2}=\omega^{2} \mathrm{~d} s^{2}(\omega>0)$, such that (i) $\left(\frac{\partial}{\partial u_{B}}\right)$ is null and affine and points along the null generators of $\mathcal{I}^{+}$, (ii) $l_{B}=\omega l+\mathcal{O}\left(l^{2}\right)$, and (iii) the conformal metric in the subspace ( $u_{B}=$ const, $l_{B}=$ const $)$ is the unit-sphere metric on $\mathcal{I}^{+}$. We fix a null tetrad on $\mathcal{I}^{+}$by choosing $n^{a}$ to be affine and to point along the null generators of $\mathcal{I}^{+}$and by choosing $m^{(a} \bar{m}^{b)}$ to be the unit-sphere covariant metric in the two-dimensional angular subspace. In inertial coordinates $\left(u_{B}, q_{B}, p_{B}, l_{B}\right)$ on $\mathcal{I}^{+}$, the tetrad has the form

$$
\begin{aligned}
& \tilde{n}^{a}=(1,0,0,0), \\
& \tilde{l}^{a}=(0,0,0,1), \\
& \tilde{m}^{a}=(0, \tilde{P} / 2, \mathrm{i} \tilde{P} / 2,0),
\end{aligned}
$$

where the tilde denotes a quantity defined with respect to inertial observers. Here, $\tilde{P}$ as $P$ introduced in equation (3.2), but for an inertial observer. Note that $\tilde{m}^{(A} \overline{\tilde{m}}^{B)}=q^{A B}$ as required. We define a complex vector $F^{a}$, analogous to $\tilde{m}^{a}$ (i.e. $F^{(A} \bar{F}^{B)}=H^{A B}$ ), adapted to the coordinates used in the characteristic evolution code via

$$
F^{a}=\left(0, F^{A}, 0\right),
$$

where

$$
F^{A}=q^{A} \sqrt{\frac{K_{0}+1}{2}}-J_{0} \bar{q}^{A} \sqrt{\frac{1}{2\left(K_{0}+1\right)}},
$$

where $q^{A}$ is the dyad defined in section $3, J_{0}=q^{A} q^{B} H_{A B} / 2$ and $K_{0}=q^{A} \bar{q}^{B} H_{A B} / 2 . F^{a}$ and $\tilde{m}^{a}$ are related by

$$
\tilde{m}^{a}=\mathrm{e}^{-\mathrm{i} \delta} \omega^{-1} F^{a}+\gamma \tilde{n}^{a} .
$$

The $\gamma \tilde{n}^{a}$ term will not enter the news calculation. 
To calculate the Bondi news, one needs to evolve two scalar quantities, $\delta$, the phase factor in equation (4.7), and the conformal factor $\omega$, as well as the relations $\xi\left(\xi_{B}\right)$ between the angular coordinates used in the characteristic evolution and inertial angular coordinates, and $u_{B}\left(u, \xi_{B}\right)$ between the inertial time slicing and the time slicing of the characteristic evolution code. Then, $\delta, \xi\left(\xi_{B}\right)$ and $u_{B}\left(u, \xi_{B}\right)$ are evolved using the following ODEs along the null generators of $\mathcal{I}^{+}$:

$$
\begin{aligned}
\frac{\mathrm{d} \delta}{\mathrm{d} u} & =\frac{1}{2} \Im\left(\frac{\bar{J}_{0, u} J_{0}}{K_{0}+1}+\frac{J_{0}\left(U_{0} \bar{\varnothing} \bar{J}_{0}+\bar{U}_{0} \rtimes \bar{J}_{0}\right)}{2\left(K_{0}+1\right)}+J_{0} \bar{\varnothing} \bar{U}_{0}+K_{0} \bar{\varnothing} U_{0}+2 U_{0} \bar{\xi}\right), \\
\frac{\mathrm{d} \xi}{\mathrm{d} u} & =\frac{1}{2}(1+\xi \bar{\xi}) U_{0} \\
\frac{\mathrm{d} u_{B}}{\mathrm{~d} u} & =\omega \mathrm{e}^{2 H}
\end{aligned}
$$

where $U_{0}=q_{A} L^{A}$. Also $\omega$ is evolved using the PDE

$$
\partial_{u} \log \omega=-\operatorname{Re}\left(\bar{U} ð \log \omega+\frac{1}{2} ð \bar{U}\right) .
$$

The Bondi news function up to a phase factor of $\mathrm{e}^{-2 \mathrm{i} \delta}$ is given by

$$
N=\frac{1}{2} \omega^{-2} \mathrm{e}^{-2 H} F^{A} F^{B}\left(\left(\partial_{u}+\mathcal{L}_{L}\right) c_{A B}-\frac{1}{2} c_{A B} D_{C} L^{C}+2 \omega D_{A}\left[\omega^{-2} D_{B}\left(\omega \mathrm{e}^{2 H}\right)\right]\right),
$$

where $D_{A}$ is the covariant derivative with respect to $H_{A B}$. The Bondi news is calculated in three steps. First, equation (4.12) is evaluated, ignoring the $\mathrm{e}^{-2 \mathrm{i} \delta}$ phase factor, as a function of the evolution coordinates $(u, \xi)$. Then, using the relation $\xi\left(\xi_{B}\right)$, the news is interpolated onto a fixed inertial angular grid (i.e. $N\left(u, \xi_{B}\right)$ ) and multiplied by the phase factor $\mathrm{e}^{-2 \mathrm{i} \delta}$ (which is only known on the inertial grid). Finally, in post-processing, the news is interpolated in time onto fixed inertial time slices (i.e. $N\left(u_{B}, \xi_{B}\right)$ ). Once the news is obtained in inertial coordinates, it can be decomposed into spin-weighted spherical harmonics.

\section{Tests}

We apply the algorithm described above for two sets of tests: non-radiative spacetimes and radiative spacetimes. In the first, non-radiative set of tests (section 5.1), we analyse Minkowski and Schwarzschild spacetimes. The Minkowski (section 5.1.1) or small perturbation of Minkowski (section 5.1.2) tests are used to show the stability of the code and the errors due to transforming between the different coordinate systems and sets of variables.

The Schwarzschild tests describe a static spherically symmetric black hole in a centred frame (section 5.1.3) and in an oscillating frame (section 5.1.4). These tests indicate the accuracy of the code in non-trivial spacetimes.

The last test (section 5.2) is a standard radiative test, namely a linearized Teukolsky wave, and indicates that CCE is a valid and accurate method in truly radiative situations.

In the non-radiative cases, the extracted gravitational wave signal should vanish identically. As this is true uniformly for all points at $\mathcal{I}^{+}$, we simplify the computation for the norms of the gravitational wave signal. When the results of the CCE are shown, the norm used is the simple $L_{2}$ norm over all grid points at $\mathcal{I}^{+}$, without any weighting by the area element. In the radiative case, we plot the real part of the extracted news waveform $\left(\partial_{t} h_{+}\right)$versus time.

The Zerilli Moncrief formalism (see [23, 24, 51] for the implementation used here) approximates the spacetime as a linearized perturbation of a spherically symmetric spacetime. Such perturbations are decomposed in terms of spherical harmonics which are then expressed 
in terms of some basis set. An example using the Regge-Wheeler set is where the even-parity metric perturbations are decomposed as

$$
\left(h_{\mu \nu}^{l m}\right)^{(e)}=\left(\begin{array}{cc|c}
\mathrm{e}^{2 a} H_{0} Y^{l m} & H_{1} Y^{l m} & \\
H_{1} Y^{l m} & \mathrm{e}^{2 b} H_{2} Y^{l m} & H_{A}^{(e)} \nabla_{c} Y^{l m} \\
\hline h_{A}^{(e)} \nabla_{c} Y^{l m} & & r^{2}\left(K Y^{l m} \gamma_{c d}+G \nabla_{d} \nabla_{c} Y^{l m}\right)
\end{array}\right) .
$$

Once the basis functions such as $G, K, h_{1}^{(e)}, H_{2}$ and $b$ are found from the decomposition, the even-parity master function $Q^{+}$can be computed from

$Q^{+}=\sqrt{\frac{2(l+2) !}{(l-2) !}} \frac{r\left[r \Lambda\left(K+\frac{1}{\mathrm{e}^{2 b}}\left(r \partial_{r} G-\frac{2}{r} h_{1}^{(e)}\right)\right)+\frac{2 r}{\mathrm{e}^{4 b}}\left(\mathrm{e}^{2 b} H_{2}-\mathrm{e}^{b} \partial_{r}\left(r \mathrm{e}^{b} K\right)\right)\right]}{\Lambda[r(\Lambda-2)+6 M]}$,

where $M$ is an approximation to the mass of the Schwarzschild background and $\Lambda=$ $(l-1)(l-2)+6 M / r$. Equivalent definitions in terms of other basis functions, alternative conventions and expressions for the odd-parity sector can be found in [52].

Using this notation where $Q^{\times}$gives the odd-parity master function, the asymptotic value for the waveforms is

$h_{+}-\mathrm{i} h_{\times}=\frac{1}{\sqrt{2} r} \sum_{l, m}\left(Q_{l m}^{+}-\mathrm{i} \int_{-\infty}^{t} Q_{l m}^{\times}\left(t^{\prime}\right) \mathrm{d} t^{\prime}\right){ }_{-2} Y^{l m}(\theta, \phi)+\mathcal{O}\left(\frac{1}{r^{2}}\right)$.

For the non-radiative cases, when we compute the wave signal from the Zerilli approximation, we use the norm

$$
\begin{aligned}
\left(\|h\|_{2}\right)^{2} & =\int\left(h_{+}-\mathrm{i} h_{\times}\right)\left(h_{+}-\mathrm{i} h_{\times}\right)^{\dagger} \mathrm{d} \Omega \\
& =\frac{1}{2 r^{2}}\left(Q_{\ell m}^{+}-\mathrm{i} q_{\ell m}^{\times}\right)\left(Q_{\ell^{\prime} m^{\prime}}^{+}-\mathrm{i} q_{\ell^{\prime} m^{\prime}}^{\times}\right)^{\dagger} \int_{-2} Y^{\ell m}{ }_{-2} Y^{\ell^{\prime} m^{\dagger} \dagger} \mathrm{d} \Omega \\
& =\frac{1}{2 r^{2}} \delta^{\ell \ell^{\prime}} \delta^{m m^{\prime}}\left(Q_{\ell m}^{+}-\mathrm{i} q_{\ell m}^{\times}\right)\left(Q_{\ell^{\prime} m^{\prime}}^{+}-\mathrm{i} q_{\ell^{\prime} m^{\prime}}^{\times}\right)^{\dagger},
\end{aligned}
$$

where $d \Omega$ is the element of solid angle. The last equality (5.5) follows from the orthogonality of the spin-weighted tensor spherical harmonics. We expect $h_{+}-\mathrm{i} h_{\times}$to vanish for all angles so this norm should be identically zero. In the following, we shall look at the spherical harmonics up to $\ell=m=7$, although in practice there is little contribution from the higher modes.

For the Teukolsky wave used here we expect all terms except for the $Q_{2,0}^{+}$term in equation (5.3) to be negligible asymptotically, which leads to a simple but tedious computation of the wave signal.

\subsection{Non-radiative spacetime tests}

5.1.1. Minkowski flat spacetime. Minkowski space in standard coordinates is evolved in the harmonic Abigel code, with the metric given analytically on all Cauchy slices. The domain extends between $x^{i} \in[-10,10]$, the extraction world-tube is placed at $r=7$ and the simulation is run until $t=10$. The coarsest simulation used $50^{3}$ points for the Cauchy grid and $35^{2} \times 31$ points for the characteristic grid. Simulations to test convergence scaled all grids by factors of 2 . For this test, extraction using the Zerilli method gives results that are identically zero, as expected.

This test indicates the level of numerical round-off error in transforming from the Cauchy variables to the Bondi variables on the world-tube. As shown in figure 1, the extracted news is small in all cases but the level of truncation error increases linearly with the number of 


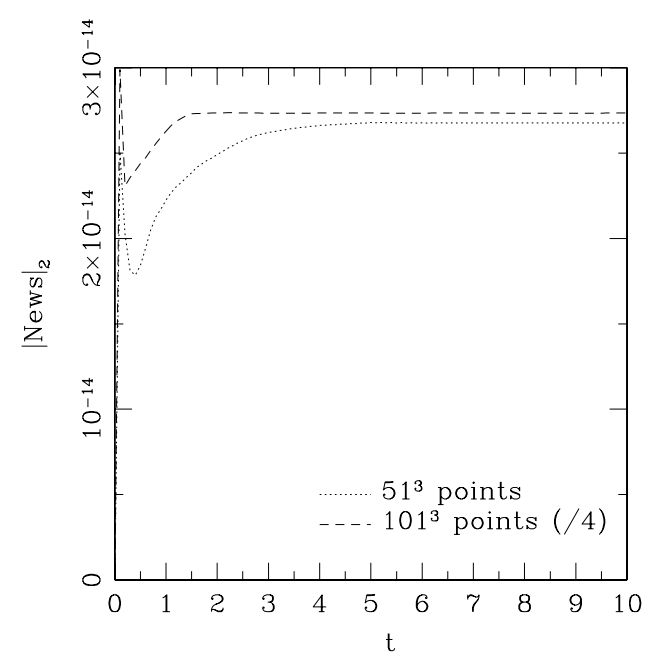

Figure 1. The $L_{2}$ norm of the CCE news for Minkowski space in standard coordinates. This indicates the level of numerical noise introduced by transforming variables on the world-tube. In this case, the noise is linearly dependent on the number of points on the extraction world-tube. It is, however, extremely small.

points on the extraction world-tube. Since the construction of the boundary data for the CCE code involves derivatives of the Cauchy metric, this sensitivity to noise in the Cauchy data is expected. However, given the extremely low amplitude of the noise, it is unlikely that this will cause problems in practical simulations.

5.1.2. Random perturbations around Minkowski flat spacetime. This test is in the spirit of the robust stability tests of $[22,67,68]$. The stable evolution and extraction of white noise initial data with no frequency dependent growth of the wave signal is a good indication that the combined evolution and extraction codes are stable. The domain extends between $x^{i} \in[-10,10]$, the extraction world-tube is placed at $r=7$ and the simulation is run until $t=100$. The coarsest Cauchy grid has $51^{3}$ points and the coarsest characteristic grid has $35^{2} \times 31$ points. The initial amplitude of the white noise initial data is $10^{-10}$ for the $50^{3}$ grid and $0.25 \times 10^{-10}$ for the $100^{3}$ grid.

The results in figure 2 show that the error is independent of the resolution of both Cauchy and characteristic grids. This is strong evidence that the CCE code is stable against small perturbations that in a practical run would be induced by numerical error. The wave signal from the Zerilli extraction is about an order of magnitude smaller. This is to be expected as the Zerilli extraction approach uses the field values. The error in the CCE news has a jump at the beginning. This arises because the initial characteristic data are set to zero so that it takes some time for the noise to build up on the outgoing null cone.

5.1.3. Schwarzschild black hole in a 'centred' frame. To test a simple black hole spacetime, we use a Schwarzschild black hole in ingoing Eddington-Finklestein coordinates $\left(\hat{t}, \hat{x}^{i}\right)$, with the line element

$$
\mathrm{d} s^{2}=-\left(1-\frac{2 M}{\hat{r}}\right) \mathrm{d} \hat{t}^{2}+\left(\frac{4 M}{\hat{r}}\right) \mathrm{d} \hat{t} \mathrm{~d} \hat{r}+\left(1+\frac{2 M}{\hat{r}}\right) \mathrm{d} \hat{r}^{2}+\hat{r}^{2} \mathrm{~d} \Omega^{2}
$$



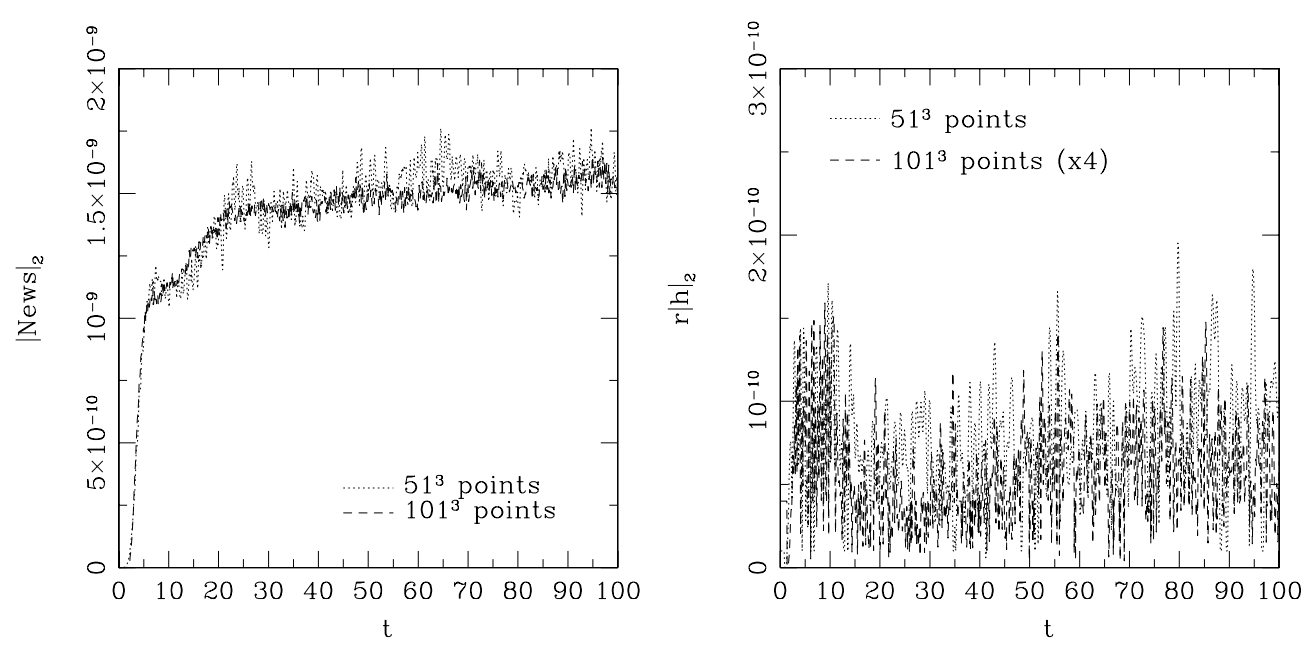

Figure 2. The $L_{2}$ norm of the CCE news for random perturbations of Minkowski where the Cauchy slice is evolved using the Abigel code. The news displays very slow growth that is independent of the frequency of the initial data. This is a good indication that the CCE method is stable. The right panel shows that the norm of the waveform extracted from the Cauchy grid using the Zerilli method is about one order of magnitude smaller.

This is manifestly static in these coordinates. The spacetime is evolved using the BSSN code and excision methods described in [69]. The coarsest Cauchy grid has $29^{3}$ points with $\Delta x^{i}=0.4 M$, whilst the characteristic grid has $35^{2} \times 31$ points. The world-tube is at $r=7 M$. In the Cauchy evolution domain octant symmetry is used.

The evolution is only performed for a short time (to $t=100 M$ ). Over this time scale, we see second-order convergence for the CCE news until $t \approx 7 M$ and second-order convergence in the waveform from Zerilli extraction until $t \approx 20 M$, as seen in figure 3 . By varying the location of the world-tube or the Zerilli extraction sphere, we can see that the errors come from a variety of locations.

The difference between the times at which the two extraction methods lose convergence is probably due to the greater differencing error seen in the Zerilli extraction. At early times (where finite-differencing error dominates), the absolute value of the error is larger for Zerilli extraction by a factor $\approx 4$, as seen in figure 3 . At late times (where outer Cauchy boundary errors dominate), the error for Zerilli extraction is of the same order as for the CCE extraction.

The non-convergent errors are probably caused by the boundary conditions on the Cauchy grid which do not satisfy the constraints. As in [69], we are simply applying Sommerfeld-type boundary conditions to all fields. This condition does not a priori satisfy the constraints and is not known to be well posed. Thus, we might expect errors to be induced by the use of these boundary conditions. It is likely that constraint satisfying boundary conditions or Cauchy-characteristic matching would solve or at least greatly reduce this problem. Due to the complicated pattern of the reflected errors arising from a boundary condition on a cubical surface, we have been unable to determine exactly how this error depends on the location of the outer Cauchy boundary.

5.1.4. Schwarzschild black hole in an oscillating frame. We use the line element for the standard Schwarzschild black hole in ingoing Eddington-Finklestein coordinates, given in equation (5.6). The moving coordinate frame $\left(t, x^{i}\right)$ is given by

$$
t=\hat{t}, \quad x^{i}=\hat{x}^{i}+B^{i} b(t),
$$



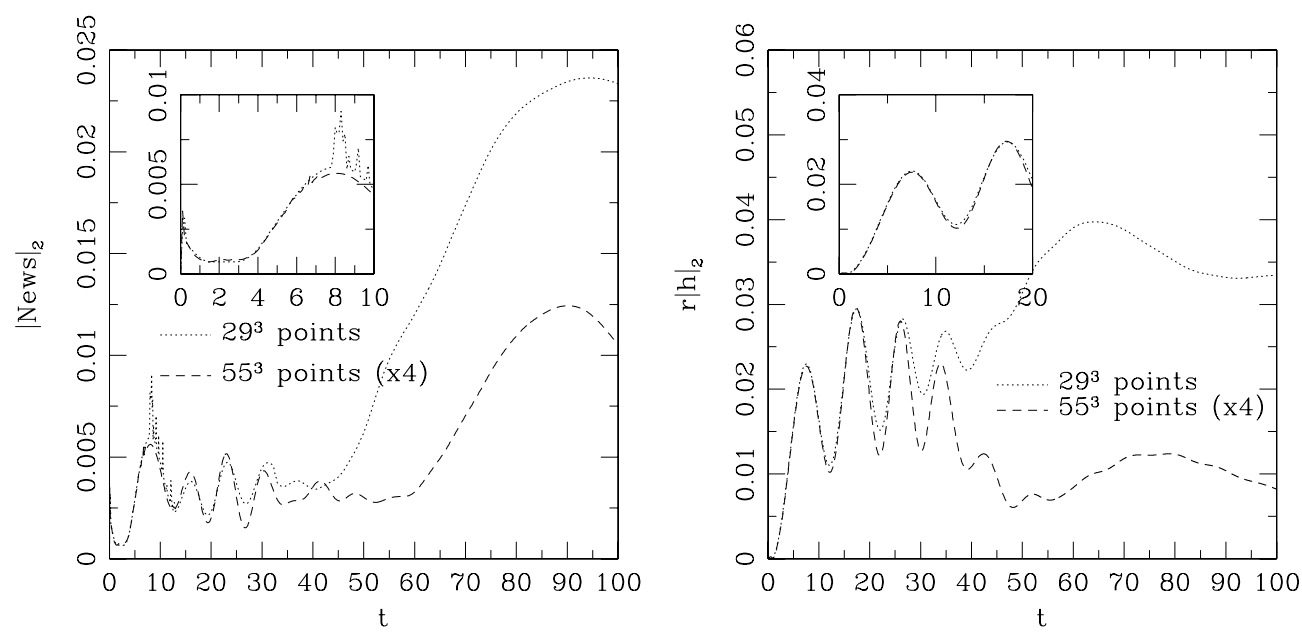

Figure 3. The scaled $L_{2}$ norm of the CCE news for a static spherically symmetric black hole where the Cauchy slice is evolved using the BSSN formalism. At early times, when the world-tube is causally disconnected from the outer boundary of the Cauchy slice, the CCE news converges to zero at second order as it should. Later, when the outer boundary is causally connected to the world-tube, deviations from second-order convergence appear, indicating the effect of the Cauchy boundary conditions on the extracted wave signal. The left panel shows the CCE news extracted at $\mathcal{I}^{+}$, whilst the right panel shows the 'norm' of the wave signal extracted by the Zerilli method at $r=7$.

where $B^{i}$ are parameters specifying the velocity and $b(t)$ is a simple periodic function turned on after some time $t>t_{0}$; here we use

$$
b(t)= \begin{cases}0, & t \leqslant t_{0}, \\ \sin \left(\omega\left(t-t_{0}\right)\right)^{3}, & t>t_{0} .\end{cases}
$$

For the test shown here, we set $B^{x}=0.2, B^{y}=0.5, B^{z}=0.3$ for the velocity and $\omega=2 \pi \times 0.05, t_{0}=0.5$ for the function $b$.

The metric is given analytically on the Cauchy grid to avoid any boundary effects. The coarsest Cauchy grid has $51^{3}$ points and covers the domain $x^{i} \in[-10 M, 10 M]$. The coarsest characteristic grid has $35^{2} \times 31$ points. The extraction world-tube is at $r=7 M$. The simulation is evolved until $t=100 \mathrm{M}$.

The CCE news converges to zero as it should. However, Zerilli extraction does not give a signal that converges to zero as grid resolution is refined. Instead, an erroneous non-trivial signal is computed.

\subsection{Teukolsky wave test}

5.2.1. Theoretical part. The Teukolsky solution [27, 53, 55] to the linearized Einstein equation is a weak gravitational wave propagating through space and represents one of the most valuable standard test cases for numerical codes that implement wave extraction techniques.

The general form of the spacetime metric is

$$
\begin{aligned}
\mathrm{d} s^{2}=-\mathrm{d} t^{2}+ & \left(1+A f_{r r}\right) \mathrm{d} r^{2}+2 B f_{r \theta} r \mathrm{~d} r \mathrm{~d} \theta+2 B f_{r \phi} r \sin \theta \mathrm{d} r \mathrm{~d} \phi \\
& +\left(1+C f_{\theta \theta}^{(1)}+A f_{\theta \theta}^{(2)}\right) r^{2} \mathrm{~d} \theta^{2}+2(A-2 C) f_{\theta \phi} r^{2} \sin \theta \mathrm{d} \theta \mathrm{d} \phi \\
& +\left(1+C f_{\phi \phi}^{(1)}+A f_{\phi \phi}^{(2)}\right) r^{2} \sin ^{2} \theta \mathrm{d} \phi^{2},
\end{aligned}
$$




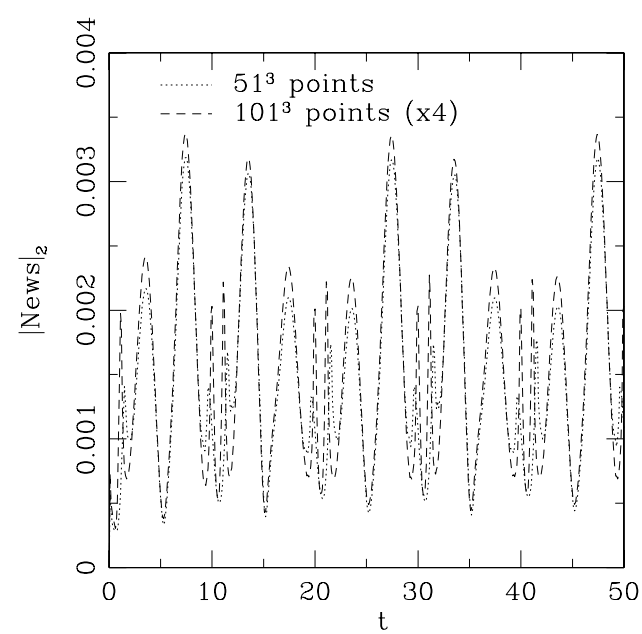

Figure 4. The scaled $L_{2}$ norm of the CCE news for a static spherically symmetric black hole in an oscillating frame. The CCE news converges to zero at second order as it should.
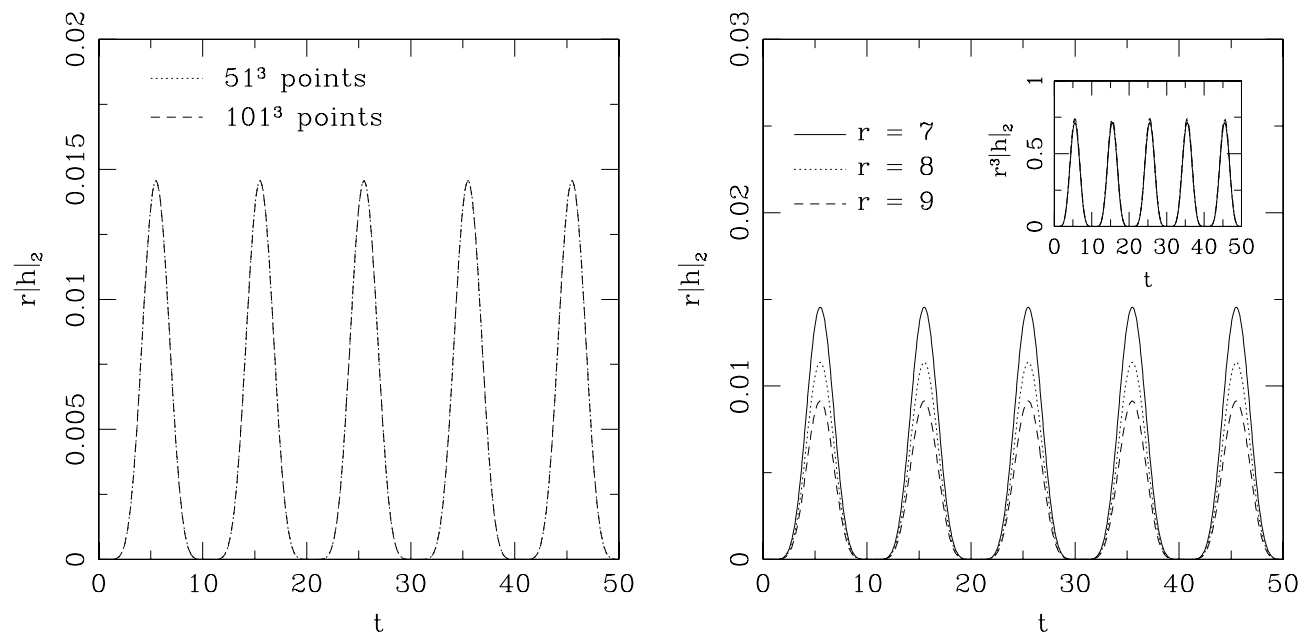

Figure 5. The wave signal computed using Zerilli extraction for the static spherically symmetric black hole in an oscillating frame. The 'norm' is computed as described in the text with the radial dependence removed. The left panel shows that as the resolution of both the Cartesian grid and the extraction sphere are increased, the signal does not converge to zero. The right panel shows how this erroneous non-trivial wave signal decays as $\mathcal{O}\left(r^{-2}\right)$ as the extraction radius is increased. In the main plot, the standard scaling of the signal is used. In the inset, the curves are scaled by the extraction radius so as to overlay each other for the expected decay rate.

where the angular functions $f_{i j}$, corresponding to an $l=2, m=0$, spin-weight $=2$ spherical harmonic, are

$$
\begin{array}{ll}
f_{r r}=2-3 \sin ^{2} \theta, & f_{r \theta}=-3 \sin \theta \cos \theta, \quad f_{r \phi}=0, \\
f_{\theta \theta}^{(1)}=3 \sin ^{2} \theta, & f_{\theta \theta}^{(2)}=-1, \\
f_{\theta \phi}=0, & f_{\phi \phi}^{(1)}=-f_{\theta \theta}^{(1)}, \quad f_{\phi \phi}^{(2)}=3 \sin ^{2} \theta-1 .
\end{array}
$$


The functions $A, B$ and $C$ are given in terms of a free generating function $F(x)=$ $\mathcal{A} x \mathrm{e}^{-x^{2} / \lambda^{2}} / \lambda^{2}$, where $\mathcal{A}$ is the amplitude and $\lambda$ determines the width of the wave, by

$$
\begin{aligned}
& A=3\left(\frac{\mathrm{d}_{x}^{2} F}{r^{3}}+\frac{3 \mathrm{~d}_{x} F}{r^{4}}+\frac{3 F}{r^{5}}\right), \\
& B=-\left(\frac{\mathrm{d}_{x}^{3} F}{r^{2}}+\frac{3 \mathrm{~d}_{x}^{2} F}{r^{3}}+\frac{6 \mathrm{~d}_{x} F}{r^{4}}+\frac{6 F}{r^{5}}\right), \\
& C=\frac{1}{4}\left(\frac{\mathrm{d}_{x}^{4} F}{r}+\frac{2 d_{x}^{3} F}{r^{2}}+\frac{9 \mathrm{~d}_{x}^{2} F}{r^{3}}+\frac{21 \mathrm{~d}_{x} F}{r^{4}}+\frac{21 F}{r^{5}}\right),
\end{aligned}
$$

where $\mathrm{d}_{x}^{n} F$ denotes

$$
\mathrm{d}_{x}^{n} F=\frac{\mathrm{d}^{n} F(x)}{\mathrm{d} x^{n}}
$$

Here, $x=t-r$ corresponds to an outgoing wave. To obtain the ingoing wave solution corresponding to $x=t+r$, we change the sign in front of all the terms with odd numbers of $F$.

We consider a superposition of ingoing $t+r$ and outgoing $t-r$ waves [44], centred at the origin of the coordinate system at $t=0$, which provides a moment of time symmetry. This solution, after is linearized in amplitude, is implemented in the harmonic Abigel code.

We further give the analytical form of the Teukolsky wave signal. The real part of the Bondi news function is proportional to the time derivative of the 'plus' polarization mode of the gravitational wave signal. The time derivative of the CCE news satisfies

$$
\dot{N}=\lim _{r \rightarrow \infty} r \Psi_{4}
$$

where $\Psi_{4}$ is the Newman-Penrose component of the Weyl tensor

$$
\Psi_{4}=-C_{\mu \rho v \tau} n^{\mu} \bar{m}^{\rho} n^{v} \bar{m}^{\tau}
$$

with $n^{v}$ an ingoing null vector and $m^{\rho}$ a complex unit vector, oriented in the angular directions.

The connection between $m^{\rho}$ and the dyad vector $q^{A}$ on the unit sphere is

$$
m^{A}=\frac{1}{r} q^{A} .
$$

Moreover, $n^{\mu} \partial_{\mu}=\partial_{x}$. A straightforward computation starting from equation (5.13) gives

$$
\dot{N}=-\frac{1}{r} \bar{q}^{A} \bar{q}^{B} \partial_{x}^{2} g_{A B}
$$

At infinity, in the linearized regime, only the $\mathcal{O}(1 / r)$ terms in the Teukolsky functions $A, B$ and $C$ contribute to the news. The term that determines the gravitational wave signal is $\mathrm{d}_{x}^{4} F$. We find

$$
\dot{N}=-\frac{3 \sin ^{2} \theta}{4} \partial_{t}^{6} F
$$

Alternatingly, computation of the linearized expression for the Bondi news from equation (4.12) gives

$$
N=-\frac{3 \sin ^{2} \theta}{4} \partial_{t}^{5} F .
$$



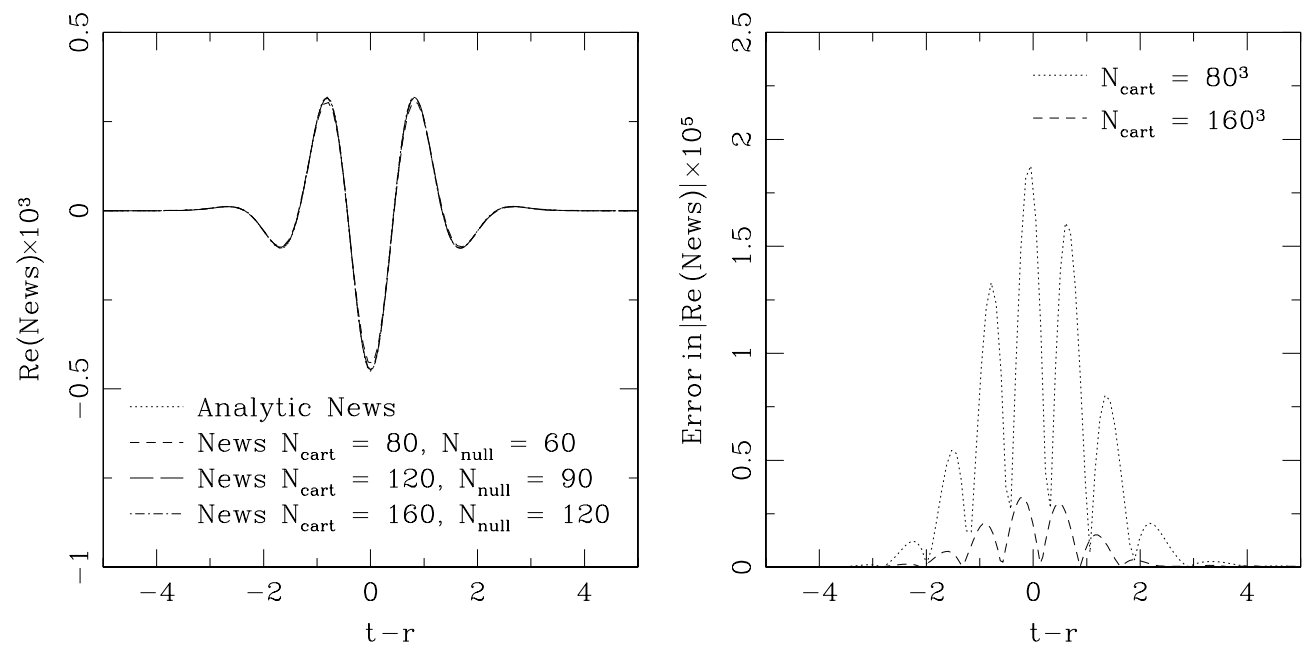

Figure 6. Left panel: the convergence of CCE news to the analytical solution. In this case, the grid resolution was (Cartesian/characteristic) $80^{3} / 60^{2} \times 80,120^{3} / 90^{2} \times 120$ and $160^{3} / 120^{2} \times 160$, respectively. Right panel: the absolute value of the error in CCE news for the grid resolutions of $80^{3} / 60^{2} \times 80$ and $160^{3} / 120^{2} \times 160$.

\subsection{Numerical part}

We give the metric specified by the Teukolsky solution analytically on the Cauchy grid. Then, data are extracted at the world-tube and evolved by the CCE code. We carry out a series of simulations, varying both the location of the characteristic world-tube and the radius of the Zerilli sphere. We use $80^{3}, 120^{3}$ and $160^{3}$ grid points for the Cartesian Cauchy grid and $60^{2} \times 80,90^{2} \times 120$ and $120^{2} \times 160$ grid points for the null grid. The domain extends between $x^{i} \in[-15,15]$ and the simulations are run until $t=30$.

We study the dependence of the signal with the amplitude and conclude that CCE code resolves correctly amplitudes of $\mathcal{A} \geqslant 10^{-8}$. At smaller amplitudes, clean convergence behaviour is contaminated by round-off error. We show results only for $\mathcal{A}=10^{-5}, \lambda=1$. We also study the dependence of the wave signal with the world-tube radius and conclude that the accuracy of the computed CCE news is preserved even for radii as small as $r=5$.

Figure 6 shows the convergence of the CCE news to the analytical solution, for a worldtube radius of $r=10$. The convergence rate $\tilde{c}_{r}$ of the CCE news to the analytical data is given by

$$
\tilde{c}_{r}=\log _{2}\left(\frac{\left\|N_{80}-N_{\text {ana }}\right\|}{\left\|N_{160}-N_{\text {ana }}\right\|}\right)
$$

The convergence rate of the computed CCE news to the analytic value (5.18) at $t-r=0$, corresponding to the peak of the radiated signal, is

$$
\tilde{c}_{r}=2.159 .
$$

Hence, the CCE wave signal is second-order convergent and independent of the world-tube radius, as expected. The convergence to the expected solution is independent of time also, as the Cauchy data are prescribed analytically, so the boundary errors present in the centred frame black hole evolution of section 5.1.3 do not arise.

Figure 7 shows that for small extraction radii, the Zerilli waveform has a slight asymmetry, which is caused by the dependence of the Zerilli formalism upon the extraction radius. We 

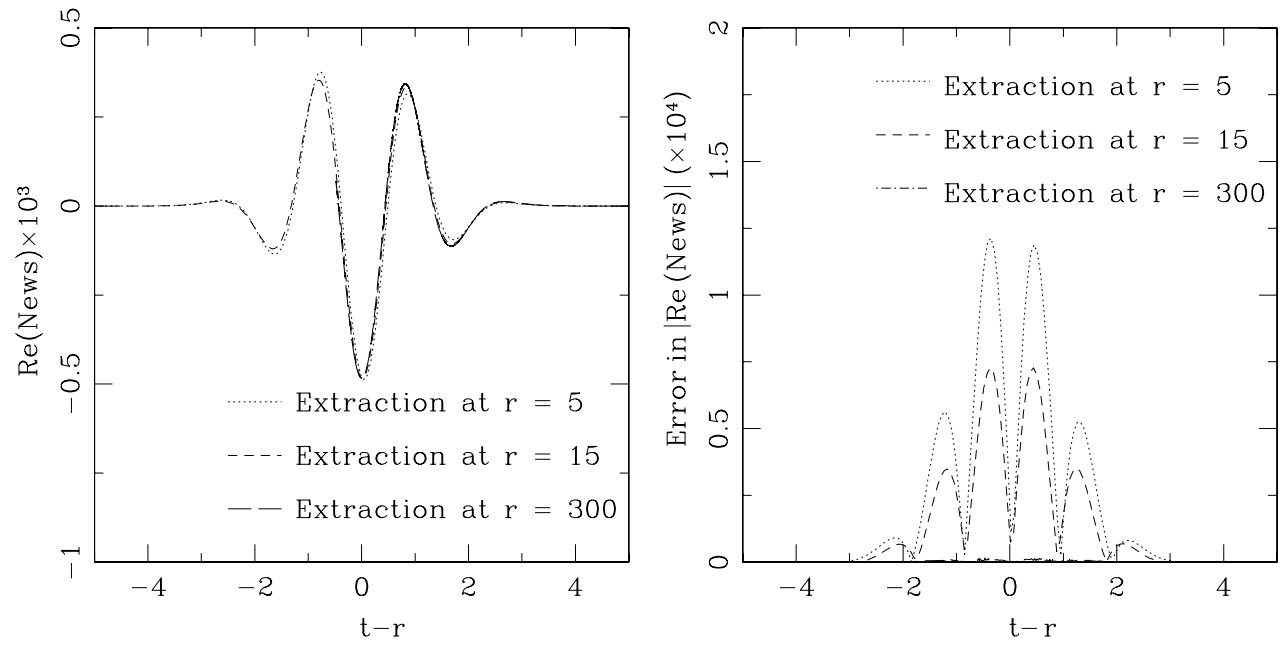

Figure 7. Left panel: the Zerilli news shows a dependence on the extraction radius. The asymmetry decreases as the extraction radius increases. Right panel: the absolute value of the error in Zerilli news with radius (grid resolution of $120^{3} / 90^{2} \times 120$ ).

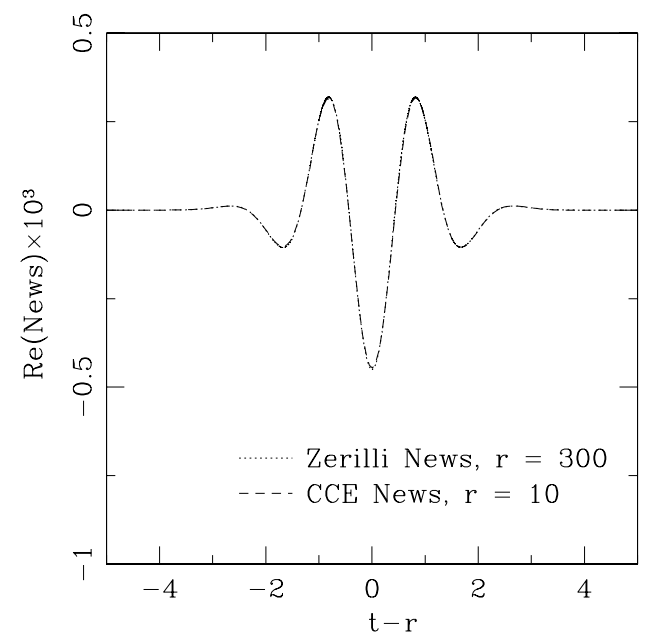

Figure 8. When the extraction radius is sufficiently large, the comparison between the Zerilli and the CCE news is in very good agreement.

have to increase the extraction radius in order to decrease this error. Because the error does not fall off sufficiently fast with radius in the near zone, where we can realistically carry out the simulation, we instead analytically compute the Zerilli news at the extraction radius $r=300$.

Figure 8 demonstrates that the agreement between the computed CCE news at small radii and the analytical Zerilli news at big radii is very good.

\section{Conclusions}

We have demonstrated the accuracy of Cauchy-characteristic extraction in 3D numerical relativity. The interface at the extraction world-tube does not introduce significant error with either the BSSN [59] or harmonic [22, 61] formulations. 
The CCE news is stable against small perturbations on the Cauchy grid, as shown in section 5.1.2, and the level of truncation error caused by transforming from the Cauchy to the characteristic variables is small, as shown in section 5.1.1. In a non-trivial black hole spacetime, CCE performs as expected. Second-order convergence is found in the moving Schwarzschild black hole test in section 5.1.4 and in the early-time behaviour of the BSSN test in section 5.1.3. However, as seen in the late-time behaviour in section 5.1.3, the effect of improper BSSN outer boundary conditions on the Cauchy grid is clearly visible in the extracted CCE news.

In the linearized Teukolsky wave test in section 5.2, we demonstrate that the computed CCE news converges to the analytic Teukolsky waveform and does not depend on the worldtube radius. The accuracy of the CCE news is preserved even for small radii, while the Zerilli news is affected by near zone error. When the extraction radius is sufficiently large, both Zerilli and CCE give excellent results. The advantage of CCE over the Zerilli method is that the extracted CCE news does not depend on the extraction radius.

Cauchy-characteristic extraction can be seen either as a first step towards a full Cauchycharacteristic matching code or as an improved gravitational wave extraction method in its own right. The results of this paper show that as a stand-alone wave extraction method, it produces the correct results in situations where other methods, such as Zerilli extraction, may fail. Such a situation is seen in section 5.1.4, where the Zerilli method fails to converge as a result of the pure gauge motion of the Schwarzschild metric.

Finally, these tests also show the need for improvement of the current implementation of the code. Any angular dependence in the solution whether through genuine physics, gauge effects or due to the imposition of boundary conditions on the cubical Cauchy boundary, leads to short wavelength error that is poorly resolved by the CCE code. This is particularly noticeable for features in the region where the stereographic patches overlap. Improvements in this area of the implementation will enhance the accuracy of the code in extracting the gravitational news from astrophysical simulations requiring high resolution. To this end, we are investigating the use of different multiple patch implementations such as [65].

\section{Acknowledgments}

We are grateful to Nigel Bishop for help at the start of this project. Also, we are grateful to Jeff Winicour for his support throughout the project as well as for his careful reading of the manuscript. Computer simulations were done at the PEYOTE cluster of the AlbertEinstein-Institut in Golm, Germany, and at the Pittsburgh Supercomputing Center under grant PHY040015P. While importing the extraction code into the Cactus infrastructure we have benefited from the support of the Cactus teams at AEI and LSU, for which we are grateful. We thank LSU for its hospitality. MB was supported by the National Science Foundation under grant PHY-0244673 to the University of Pittsburgh. BSz was partially supported by the National Science Foundation under grant PHY-0244673 to the University of Pittsburgh. IH was partially supported by PPARC grant PPA/G/S/2002/00531. YZ was partially supported by the NASA Center for Gravitational Wave Astronomy at The University of Texas at Brownsville (NAG5-13396) and by NSF grants PHY-0140326 and PHY-0354867.

\section{References}

[1] Hübner P 2001 Class. Quantum Grav. 181871

[2] Frauendiener J 2004 Living Rev. Rel. 71 http://www.livingreviews.org/lrr-2004-1 
[3] Husa S 2003 Proc. 2001 Spanish Relativity Meeting (Lecture Notes in Physics vol 617) ed L Fernández and L M González (Berlin: Springer) pp 159-92

[4] Friedrich H 1981 Proc. R. Soc. A 375169

[5] Friedrich H 1981 Proc. R. Soc. A 378401

[6] Fodor G and Rácz I 2004 Phys. Rev. Lett. 92151801

[7] Gómez R, Winicour J and Schmidt B 1994 Phys. Rev. D 492828

[8] Hamadé R S and Stewart J M 1996 Class. Quantum Grav. 13497 (Preprint gr-qc/9506044)

[9] Garfinkle D 1995 Phys. Rev. D 515558

[10] Husa S, Lechner C, Pürrer M, Thornburg J and Aichelburg P C 2000 Phys. Rev. D 62104007 (Preprint grqc/0002067)

[11] Husain V and Olivier M 2001 Class. Quantum Grav. 18 L1

[12] Husain V, Kunstatter G, Preston B and Birukou M 2003 Class. Quantum Grav. 20 L23

[13] Gnedin M L and Gnedin N Y 1993 Class. Quantum Grav. 101083

[14] Brady P R and Smith J D 1995 Phys. Rev. Lett. 751256

[15] Burko L M 1997 Phys. Rev. Lett. 794958

[16] Hod S and Piran T 1998 Phys. Rev. Lett. 811554

[17] Bishop N T, Gómez R, Lehner L, Maharaj M and Winicour J 1999 Phys. Rev. D 60024005

[18] Siebel F, Font J A, Müller E and Papadopoulos P 2002 Phys. Rev. D 65064038

[19] Siebel F, Font J A, Mueller E and Papadopoulos P 2003 Preprint gr-qc/0301127 (Phys. Rev. D submitted)

[20] Gómez R, Marsa R and Winicour J 1997 Phys. Rev. D 566310 (Preprint gr-qc/9708002)

[21] Bishop N, Gómez R, Holvorcem P, Matzner R, Papadopoulos P and Winicour J 1997 J. Comput. Phys. 136140

[22] Szilagyi B, Schmidt B and Winicour J 2002 Phys. Rev. D 65064015 (Preprint gr-qc/0106026)

[23] Zerilli F J 1970 Phys. Rev. Lett. 24737

[24] Moncrief V 1974 Ann. Phys., NY 88323

[25] Rezzolla L, Abrahams A M, Baumgarte T W, Cook G B, Scheel M A, Shapiro S L and Teukolsky S A 1998 Phys. Rev. D 571084

[26] Shibata M, Taniguchi K and Nakamura T 1997 Prog. Theor. Phys. Suppl. 128295 (Preprint gr-qc/9801004)

[27] Nakao K, Harada T, Shibata M and Kawamura S 2001 Phys. Rev. D 63082001

[28] Yo H-J, Baumgarte T and Shapiro S L 2001 Phys. Rev. D 63064035

[29] Baiotti L, Hawke I, Rezzolla L and Schnetter E 2005 Phys. Rev. Lett. 94131101 (Preprint gr-qc/0503016)

[30] Sperhake U, Kelly B, Laguna P, Smith K L and Schnetter E 2005 Preprint gr-qc/0503071

[31] Rupright M E, Abrahams A M and Rezzolla L 1998 Phys. Rev. D 58044005

[32] Rezzolla L, Abrahams A M, Matzner R A, Rupright M and Shapiro S L 1999 Phys. Rev. D 59064001

[33] Abrahams A M et al 1998 Phys. Rev. Lett. 801812 (Preprint gr-qc/9709082)

[34] Bishop N T, Isaacson R, Gómez R, Lehner L, Szilagyi B and Winicour J 1998 On the Black Hole Trail ed B Iyer and B Bhawal (Dordrecht: Kluwer) (Preprint gr-qc/9801070)

[35] Bishop N T, Gómez R, Lehner L and Winicour J 1996 Phys. Rev. D 546153

[36] Lehner L 1999 J. Comput. Phys. 14959

[37] Lehner L 1998 PhD Thesis University of Pittsburgh

[38] Gómez R, Lehner L, Marsa R and Winicour J 1998 Phys. Rev. D 574778 (Preprint gr-qc/9710138)

[39] Bishop N T, Gómez R, Lehner L, Maharaj M and Winicour J 1997 Phys. Rev. D 566298 (Preprint gr-qc/ 9708065)

[40] Gómez R 2001 Phys. Rev. D 64024007

[41] Winicour J 1998 Living Rev. Rel. 15 http://www.livingreviews.org/lrr-1998-5 (update lrr-2001-3)

[42] Winicour J 2005 Preprint gr-qc/00508097 (Living Rev. Rel. submitted)

[43] Bishop N, Isaacson R, Gómez R, Lenher L, Szilágyi B and Winicour J 1999 Black Holes, Gravitational Radiation and the Universe ed B Iyer and B Bhawal (Dordrecht: Kluwer) p 393

[44] Szilágyi B 2000 PhD Thesis University of Pittsburgh

[45] Zlochower Y 2002 PhD Thesis University of Pittsburgh

[46] Zlochower Y, Gómez R, Husa S, Lenher L and Winicour J 2003 Phys. Rev. D 68084014

[47] Allen G, Benger W, Goodale T, Hege H, Lanfermann G, Merzky A, Radke T, Seidel E and Shalf J 2001 Cluster Comput. 4179 http://www.cactuscode.org/Papers/CactusTools.ps.gz, http://www.cactuscode.org/ Papers/CactusTools.ps.gz

[48] Allen G, Benger W, Dramlitsch T, Goodale T, Hege H, Lanfermann G, Merzky A, Radke T and Seidel E 2001 Europar 2001: Parallel Processing, Proc. 7th Int. Conf. (Manchester, UK, 28-31 August 2001) ed R Sakellariou, J Keane, J Gurd and L Freeman (Berlin: Springer) http://www.cactuscode.org/Papers/ Europar01.ps.gz

[49] Cactus Code Toolkit http://www.cactuscode.org 
[50] Goodale T, Allen G, Lanfermann G, Massó J, Radke T, Seidel E and Shalf J 2003 Vector and Parallel Processing_VECPAR'2002, 5th Int. Conf. (Lecture Notes in Computer Science) (Berlin: Springer)

[51] Camarda K and Seidel E 1999 Phys. Rev. D 59064019 (Preprint gr-qc/9805099)

[52] Nagar A and Rezzolla L 2005 Class. Quantum Grav. 22 R167

[53] Kidder L E, Scheel M A and Teukolsky S A 2001 Phys. Rev. D 64064017 (Preprint gr-qc/0105031)

[54] Bonazzola S, Gourgoulhon E, Grandclement P and Novak J 2004 Phys. Rev. D 70104007 (Preprint grqc/0307082)

[55] Fiske D R, Baker J G, van Meter J R, Choi D and Centrella J M 2005 Phys. Rev. D 71104036 (Preprint gr-qc/0503100)

[56] Arnowitt R, Deser S and Misner C W 1962 Gravitation: An Introduction to Current Research ed L Witten (New York: Wiley) pp 227-65 (Preprint gr-qc/0405109)

[57] Shibata M and Nakamura T 1995 Phys. Rev. D 525428

[58] Baumgarte T W and Shapiro S L 1999 Phys. Rev. D 59024007 (Preprint gr-qc/9810065)

[59] Alcubierre M, Brügmann B, Diener P, Koppitz M, Pollney D, Seidel E and Takahashi R 2003 Phys. Rev. D 67084023 (Preprint gr-qc/0206072)

[60] Wald R M 1984 General Relativity (Chicago, IL: University of Chicago Press)

[61] Szilagyi B and Winicour J 2002 Phys. Rev. D 68041501 (Preprint gr-qc/0205044)

[62] Bondi H, Sachs F, van der Burg M G J and Metzner A W K 1962 Proc. R. Soc. A 26921

[63] Sachs R 1962 Proc. R. Soc. A 270103

[64] Gómez R, Lehner L, Papadopoulos P and Winicour J 1997 Class. Quantum Grav. 14977 (Preprint gr-qc/ 9702002)

[65] Thornburg J 2004 Class. Quantum Grav. 213665 (Preprint gr-qc/0404059)

[66] Bishop N and Deshingkar S 2003 Phys. Rev. D 68024031

[67] Szilágyi B, Gómez R, Bishop N T and Winicour J 2000 Phys. Rev. D 62104006 (Preprint gr-qc/9912030)

[68] Alcubierre M et al 2004 Class. Quantum Grav. 21589 (Preprint gr-qc/0305023)

[69] Alcubierre M and Brügmann B 2001 Phys. Rev. D 63104006 (Preprint gr-qc/0008067) 